\title{
THE EMPLOYMENT OF UV-SPECTROSCOPY COMBINED WITH MULTIVARIATE CALIBRATION FOR ANALYSIS OF PARACETAMOL, PROPYPHENAZONE AND CAFFEINE
}

\begin{abstract}
Arief Dzulfianto ${ }^{1}$, Florentinus Dika Octa Riswanto ${ }^{1}$ and Abdul Rohman ${ }^{2 *}$
1Faculty of Pharmacy, Sanata Dharma University, Yogyakarta, Indonesia. 2Department of Pharmaceutical Chemistry, Faculty of Pharmacy, Universitas Gadjah Yogyakarta 55281, Indonesia.

Submitted: 08-10-2017

Revised: $15-11-2017$

Accepted: $20-12-2017$

*Corresponding author Abdul Rohman

Email:

abdul_kimfar@ugm.ac.id

ABSTRACT

The reference method for simultaneous analysis of drugs is chromatography, however, this technique is expensive, complex, and needs excessive sample preparation; therefore, some simple methods like UV spectroscopy is proposed. Assisted with multivariate calibration, it is possible to analyze drugs using UV spectroscopy without prior separation. This study is intended to use UV spectroscopy coupled with multivariate calibration of partial least square (PLS) for simultaneous analysis of paracetamol (PCT), propyphenazone (PROPI), and caffeine (CAFF) in tablet dosage form. The calibration model was prepared by developing a series 20 mixture of PCT, PROPI and CAFF with certain composition randomly and its absorbance was measured at wavelength of $220-313 \mathrm{~nm}$ with an interval of $3 \mathrm{~nm}$. The performance of calibration model was assessed by coefficient of determination $\left(R^{2}\right)$, root mean square error of calibration (RMSEC) and root mean square error of cross validation (RMSECV). The $R^{2}$ values for the correlation between actual values of PCT, PROPI and CAFF and predicted values using UV-spectroscopy combined with PLS were $0.9994 ; 0.9878$; and 0.9919 , respectively. The calibration errors expressed with RMSEC were $0.027 \%, 0.082 \%$ and $0.043 \%$ for PCT, PROPI and CAFF, respectively. While, during cross validation using "leave one out" technique, RMSECV values obtained were $0.062 \%, 0.095 \%$ and $0.982 \%$, respectively for PCT, PROPI and CAFF. The level of drugs obtained were $226.76 \pm 14.49 \mathrm{mg} /$ tablet (equivalent to $90.70 \%$ from labeled claim) for PCT, $135.74 \pm 11.23 \mathrm{mg} /$ tablet (equivalent to $90.49 \%$ from labeled claim) for PROPI and $51.69 \pm 2.35 \mathrm{mg} /$ tablet (equivalent to $103.38 \%$ from labeled claim) for CAFF.
\end{abstract}

Keywords: UV-spectrophotometry, multivariate calibration, partial least square, paracetamol, caffeine, and Propyphenazone.

\section{INTRODUCTION}

The drug formulations containing three active components are intended to enhance the pharmaceutical effects of each substance and to cover a larger medical treatment. Pharmaceutical dosage form containing paracetamol (PCT), propyphenazone (PROPI) and caffeine (CAFF) are used as analgesic and antipyretic which have been reported to have synergetic effect. These mixture are more effective than paracetamol, ibuprophen, and aspirin given individually (Kiersch and Minic, 2002; Soponar et al., 2013). The chemical structures of PCT, PROPI and CAFF (Figure 1).

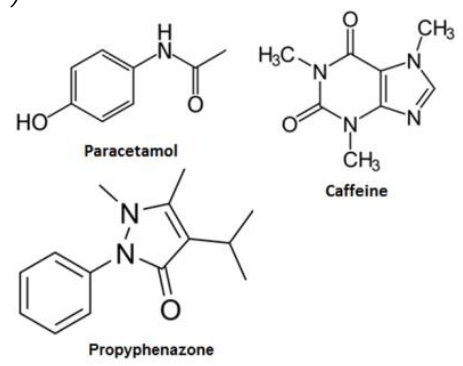

Figure 1. The chemical structures of paracetamol, propyphenazone and caffeine 
Analysis of drugs in the mixture is performed using chromatographic techniques due to the capability of chromatography to separate and to quantify the analyte(s) of interest. HPLC using diode-array detector has been used for analysis PCT, PROPI and CAFF in tablet (Soponar et al., 2013). Micellar electrokinetic capillary chromatography (Emre and Ozaltin, 2007), thin layer chromatography (Dimitrovskat et al., 1995), and gas chromategraphy (Markoviz and Kusez, 1992) have also used for analysis of PCT, PROPI and CAFF in the pharmaceuticals. However, chromatographic and electrophoretic techniques involve time consuming, skillful analyst, organic reagents and involving excessive sample preparation. Therefore, a simple and rapid technique is developed for analysis of drugs in the complex mixture without separation step such as UV spectroscopy. The use of UV spectroscopy for analysis of complex mixture is possible with the aid of several Chemometrics techniques (Rohman, 2012).

Spectrophotometry, either normal or derivative spectra, combined with some calibration techniques such as partial least square (PLS) has been used for analysis of PCT, PROPI and CAFF in two or more combinations, some with other drugs (Dinc and Onur, 1995; Dinc, 1999; Bozdogan et al., 1992). A multiparameter-responding flowthrough system with solid phase UV spectrophotometric detection (a multiparameter optosensor) has also been used for analysis of PCT, PROPI and CAFF in pharmaceuticals (Domínguez Vidal et al., 2003). However, analysis of these three drugs using UV spectrophotometry combined with PLS has not been reported yet. Therefore, in this study, the spectroscopy UV in combination with PLS is employed for quantitative analysis of PCT, PROPI and CAFF in tablet dosage forms.

Partial least square (PLS) calibration is one of the inverse calibration (Rohman, 2012), and is calculated using least squares algorithms. PLS is intended to establish a linear correlation between two matrices, the spectral data $\mathrm{X}$ and the reference or actual values of Y. In PLS, the matrices $\mathrm{X}$ and $\mathrm{Y}$ are modeled in order to find out the variables in $\mathrm{X}$ matrix capable of describing the Y matrix (Wold et al., 2001). PLS is the calibration technique widely used in analysis of multicomponent in pharmaceuticals as reviewed by Roggo et al. (2007).

\section{MATERIALS AND METHODS}

Paracetamol (PCT) was obtained from PT. Combiphar (Jakarta, Indonesia), while propyphenazone (PROPI) and caffeine (CAFF) were kindly given by PT. Konimex (Surakarta, Indonesia). The tablet used is labeled to contain 250 PCT, 150mg PROPI, and 50mg CAFF and purchased from pharmacy in Yogyakarta, Indonesia. The chemicals and reagents used were of pro analytical grade.

\section{Instrumentation and Software}

Spectrophotometer UV-Vis (Shimadzu, Japan) type UV 1800 equipped with quartz cuvette $1 \mathrm{~cm}$ (Hellma, USA) was used for UV spectra acquisition. The UV spectral data were exported to Excel (Microsoft Inc., USA) and manipulated using Minitab software version 16 (Minitab Corp., USA). All measurements were performed using $1 \mathrm{~cm}$ quartz cells over the wavelength range of $200-400 \mathrm{~nm}$ with $3 \mathrm{~nm}$ interval.

\section{Preparation of calibration standards}

The standard solutions were prepared freshly. In three separate volumetric flasks $100 \mathrm{~mL}$, an approximately of $50 \mathrm{mg}$ of each drugs (PCT, PROPI and CAFF) is accurately weighed using semi micro balance (sensitivity $0.01 \mathrm{mg}$ ), dissolved in $5 \mathrm{~mL}$ ethanol and made to volume with distilled water to obtain stock solution $50 \mathrm{mg}$ drug $/ 100 \mathrm{~mL}$. The stock solution is used for preparing calibration samples (20 samples) composed randomly using Excel (Microsoft Inc., USA), (Table I). Some synthetic mixtures with certain concentrations were also prepared to be used in validation samples (10 samples). Each solution mixture was scanned using UV spectrophotometer at 200-400nm. Each 3nm, their absorbance were recorded and used for the optimization of calibration models.

\section{Analysis of PCT, PROPI and CAFF in tablet dosage forms using UV spectrophotometry}

Twenty tablets were taken and subjected to weighting for homogeneity test. The tablets are crushed until homogenous. In volumetric flask $100 \mathrm{~mL}$, an amount of powder equivalent 
to one tablet is taken and dissolved with $10 \mathrm{~mL}$ ethanol and made to volume. The solution is shaken vigorously for $30 \mathrm{~min}$, filtered using Whatman paper, and the supernatant is taken and subjected to spectrophotometric measurement as described above. The concentration of PCT, PROPI and CAFF in tablet dosage forms is calculated based on the optimized calibration model. All determinations were performed in six times.

\section{Data analysis}

The levels of PCT, PROPI and CAFF in tablet dosage is calculated with the aid of multivariate calibration of Partial Least Square (PLS). The PLS analysis is carried out using Minitab software version 16 (Minitab Corp., USA). The correlation between actual values of these drugs and predicted values using UV spectrophotometry in combination with PLS is performed using Excel (Microsoft Inc., USA).

\section{RESULTS AND DISCUSSION}

Figure 2 revealed the overlay of UV spectra of paracetamol (PCT), propyphenazone (PROPI) and caffeine (CAFF) scanned at UV region (200-400nm). These spectra showed an extensive overlapping which limited the determination of these drugs using UV spectroscopy, simultaneously. Fortunately, some chemometrics techniques like multivariate calibration of partial least square (PLS) are developed to overcome this problem (Mohamed and Mikre, 2009; Rohman et al., 2015). Analysis of drugs in multicomponent system aided with multivariate calibration is done in three separate steps, namely calibration, validation, and analysis of unknown samples.

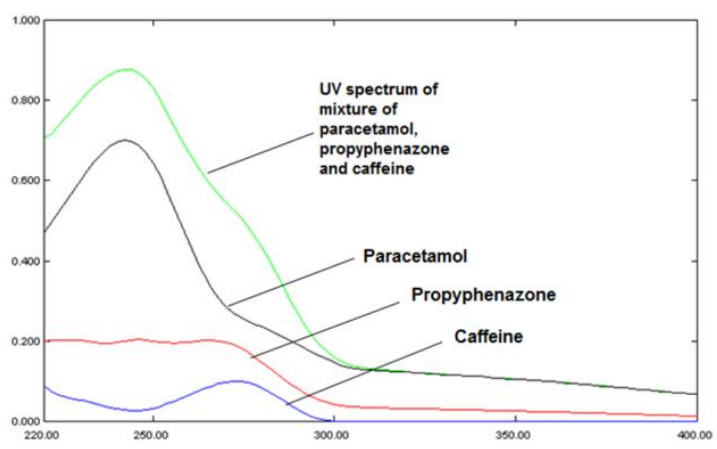

Figure 2. The UV spectra of paracetamol, caffeine, and propyphenazone as well as the mixture of three drugs, scanned at wavelength of $200-400 \mathrm{~nm}$.
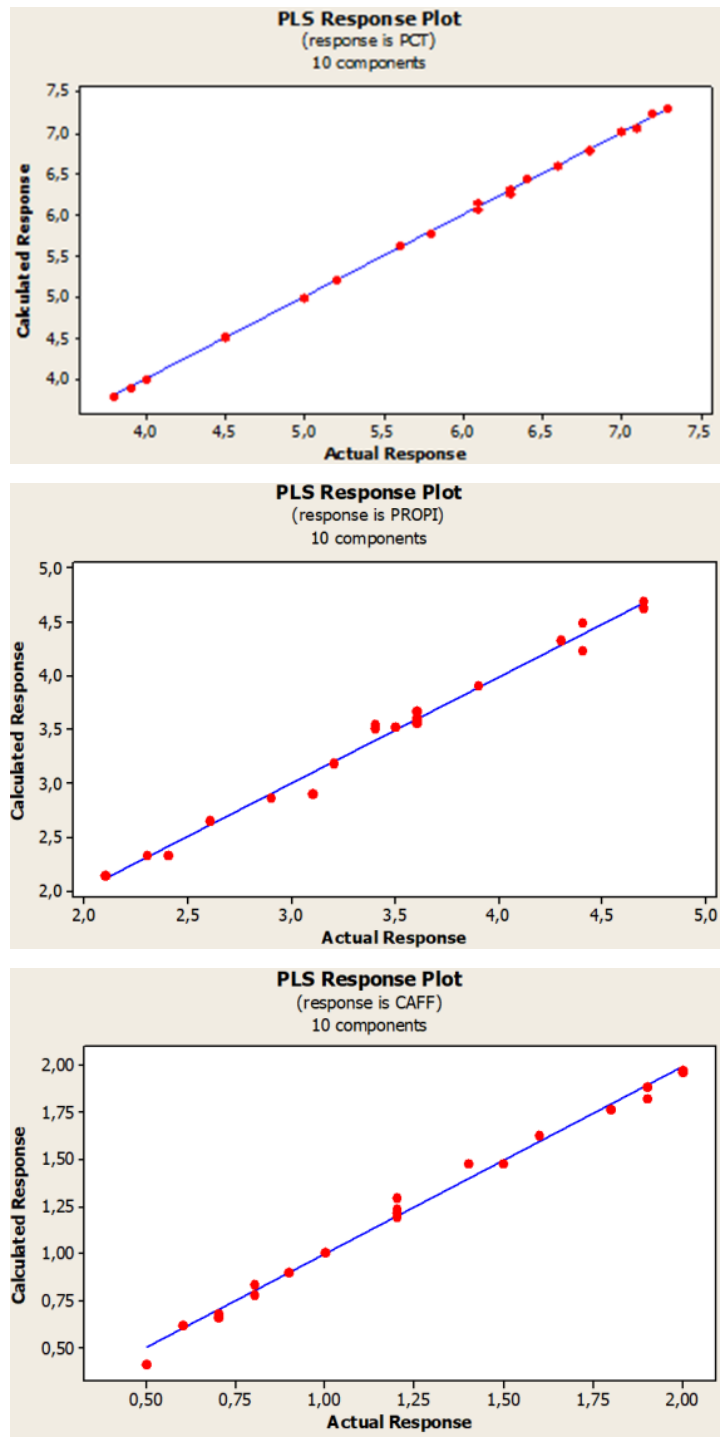

Figure 3. The PLS calibration model for the relationship between actual values of paracetamol (above), propyphenazon (middle), caffeine (below) and UV-spectroscopy predicted values.

The first step is to optimize the wavelength capable of providing the best calibration model, which offer the good correlation between actual values of PCT, PROPI and CAFF and predicted values using UV spectroscopy-PLS expressed by high value of coefficient of determination $\left(\mathrm{R}^{2}\right)$. The $\mathrm{R}^{2}$ value can be used as validation for parameter of accuracy, because $\mathrm{R}^{2}$ describes the closeness of 


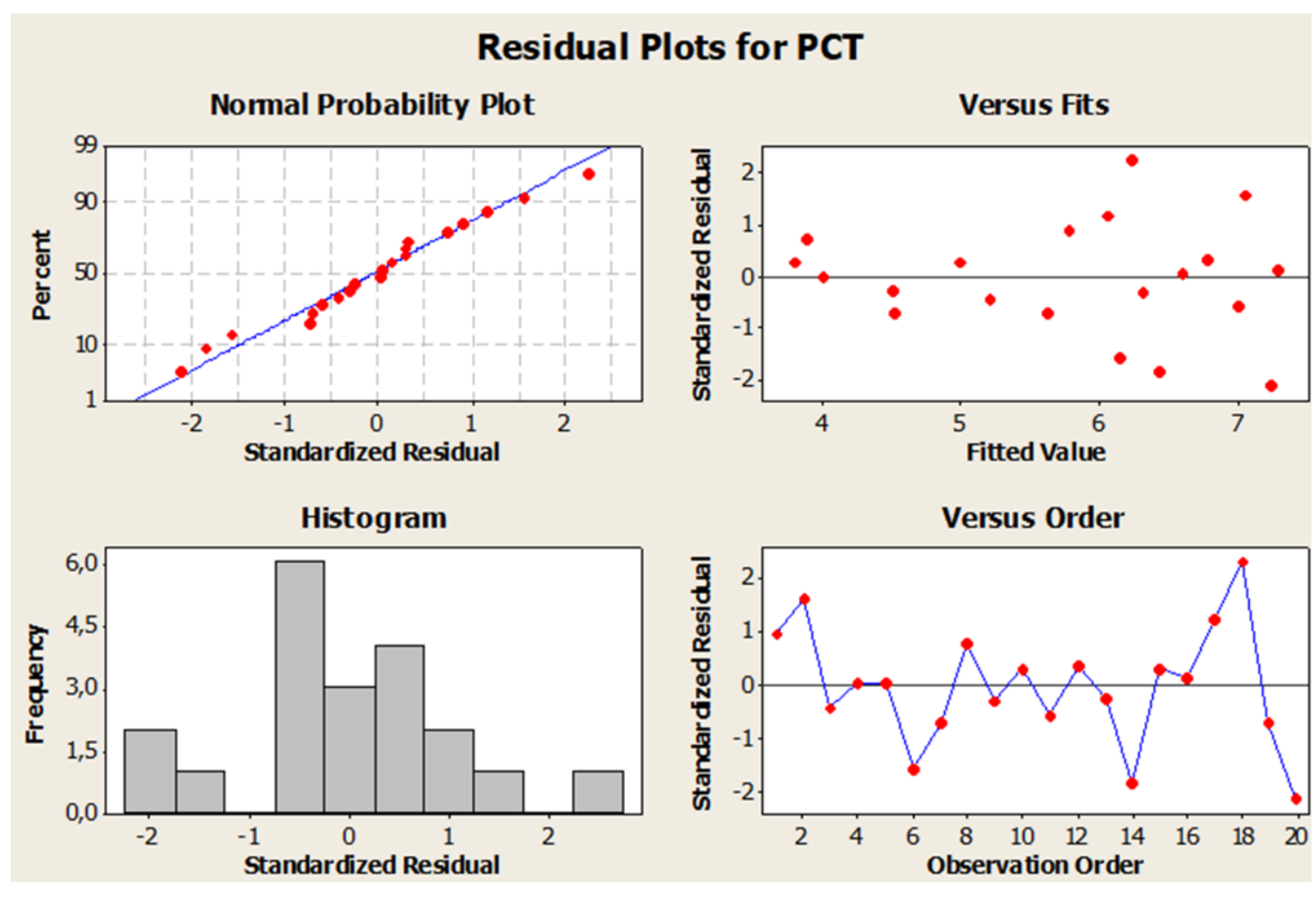

Figure 4. The residual plot of paracetamol describing the difference between actual values and predicted values of paracetamol

the predicted value with the actual value. In addition, the wavelength used during analysis is selected based on its capability to give the lowest errors, either in calibration or validation, expressed with root mean square error of calibration (RMSEC) and root mean square error of cross validation (RMSECV). RMSEC and RMSECV can be used as precision parameters, because these values describes the degree of agreement among predicted values results, when the procedure is applied repeatedly to multiple calibration samples. Based on that criteria, the wavelength of 220$313 \mathrm{~nm}$ was finally chosen for quantitative analysis of these three drugs (PCT, PROPI and CAFF) simultaneously due to its capability provide the highest values of $\mathrm{R}^{2}$ and the lowest values of RMSEC and RMSECV.

The equation used for the correlation between actual values (x-axis) and predicted values (y-axis) along with $\mathrm{R}^{2}$, RMSEC, and

RMSECV values is compiled (Table II). Figure 3 revealed the calibration model of PCT,
PROPI and CAFF using PLS regression. In addition, to figure out the random errors occurring during the calibration model, an example of the residual profile of PAR was provided (Figure 4). It is clear that random error occurred, while systematic error is negligible because the residual point existed above and below of target value (zero difference between actual and predicted value).

The calibration model is further subjected to validation using external validation technique. During external calibration, a set samples (10 samples) used as validation samples. The acceptance criteria of external calibration was based on $\mathrm{R}^{2}$ for correlation between actual value and predicted values in validation samples and RMSECV values. The high value of $\mathrm{R}^{2}$ and low values of RMSECV (Table III) indicated that the calibration model is suitable for determination of PCT, PROPI and $\mathrm{CAFF}$ in unknown samples provided that the samples contain the same drugs in the calibration and validation drug samples. 
Table I. Composition of synthetic mixture consisting of Paracetamol (PCT), Propyphenazone (PROPI), and caffeine (CAF) in calibration standard.

\begin{tabular}{cccc}
\hline No & PCT $(\boldsymbol{\mu g} / \mathbf{m L})$ & PROPI $(\boldsymbol{\mu g} / \mathbf{m L})$ & CAFF $(\boldsymbol{\mu g} / \mathbf{m L})$ \\
\hline 1 & 5.8 & 3.5 & 1.5 \\
2 & 7.1 & 3.1 & 1.2 \\
3 & 5.2 & 3.4 & 0.5 \\
4 & 6.6 & 4.7 & 0.6 \\
5 & 4.0 & 4.3 & 0.7 \\
6 & 6.1 & 3.6 & 0.8 \\
7 & 5.6 & 4.4 & 2.0 \\
8 & 3.9 & 3.9 & 0.9 \\
9 & 6.3 & 2.1 & 1.8 \\
10 & 3.8 & 2.3 & 1.0 \\
11 & 7.0 & 3.6 & 2.0 \\
12 & 6.8 & 2.9 & 1.9 \\
13 & 4.5 & 4.4 & 1.4 \\
14 & 6.4 & 2.6 & 0.7 \\
15 & 5.0 & 3.6 & 1.2 \\
16 & 7.3 & 16 & 1.6 \\
17 & 6.1 & 2.4 & 0.8 \\
18 & 6.3 & 4.7 & 1.2 \\
19 & 4.5 & 3.6 & 1.2 \\
20 & 7.2 & 3.4 & 1.9 \\
\hline
\end{tabular}

Table II The equation, coefficient of determination $\left(\mathrm{R}^{2}\right)$ and root mean square of calibration (RMSEC) for paracetamol (PCT), popiphenazone (PROPI) and caffeine (CAFF).

\begin{tabular}{lccc}
\hline & PCT & PROPI & CAFF \\
\hline Equation & $\mathrm{y}=0.9994 \mathrm{x}+0.0034$ & $\mathrm{y}=0.9878 \mathrm{x}+0.0426$ & $\mathrm{y}=0.9919 \mathrm{x}+0.0101$ \\
$\mathrm{R}^{2}$ & 0.9994 & 0.9878 & 0.9919 \\
RMSEC & 0.027445698 & 0.082897315 & 0.043408954 \\
\hline
\end{tabular}

Table III The equation, coefficient of determination $\left(\mathrm{R}^{2}\right)$, root mean square error of cross validation (RMSECV) and predicted residual error sum squares (PRESS) for paracetamol (PCT), popiphenazone (PROPI) and caffeine (CAFF).

\begin{tabular}{lccc}
\hline & PAR & PROPI & CAFF \\
\cline { 2 - 4 } Equation & $\mathrm{y}=1.0125 \mathrm{x}-0.071$ & $\mathrm{y}=0.9919 \mathrm{x}+0.0358$ & $\mathrm{y}=0.9718 \mathrm{x}+0.035$ \\
$\mathrm{R}^{2}$ & 0.993 & 0.931 & 0.940 \\
RMSECV & 0.0620 & 0.0954 & 0.0630 \\
PRESS & 0.156 & 0.782 & 0.279 \\
\hline
\end{tabular}

The final step of analysis using PLS calibration model is the evaluation of overfitting using cross validation technique. Overfitting of multivariate calibration is understood that the calibration model can generate good model in the calibration data sets, but it fails to provide optimistic model in the validation samples (Miller and Miller, 2005). During cross-validation with "leave-one out" technique, one of the calibration samples is removed from PLS model, and the remaining samples are used to make PLS model. 
Furthermore, the left out sample is calculated using the new developed PLS model. This procedure was repeated; leaving each calibration sample out in turn. Then, the difference between the actual and predicted value for each samples is computed and is expressed as root mean square error of cross validation. The sum of the squares of these differences is known as PRESS (predicted residual error sum of squares). The smaller the RMSECV and PRESS values, the better the predictive power of the calibration model (Rohman and Man, 2011). Table III also compiled the parameter values of external validation for PCT, PROPI and CAFF using PLS models.

The developed method was further used for determination of tablet formulation containing PCT, PROPI and CAFF. The level of drugs obtained are $226.76 \pm 14.49 \mathrm{mg} /$ tablet (equivalent to $90.70 \%$ from labeled claim) for PCT, $135.74 \pm 11.23 \mathrm{mg} /$ tablet (equivalent to 90.49\% from labeled claim) for PROPI, and $51.69 \pm 2.35 \mathrm{mg} /$ tablet (equivalent to $103.38 \%$ from labeled claim) for CAFF. This result confirms that UV spectroscopy in combination with multivariate calibration of PLS is an alternative method compared to reference method (high performance liquid chromatography) for simultaneous analysis of PCT, PROPI and CAFF in tablets containing these three components.

\section{CONCLUSION}

Based on the high value of $\mathrm{R}^{2}$ as well as low values of RMSEC and RMSECV, it can be concluded that UV spectroscopy coupled with multivariate calibration of PLS is an alternative techniques for simultaneous determination of PCT, PROPI and CAFF in tablet containing these drugs without any separation procedure. The method is rapid and promising for fixed formula. However, to be used in another formulation, this model should be redeveloped

\section{ACKNOWLEDGEMENT}

The authors thank to Faculty of Pharmacy for financial support during this study. We also thank to PT. Combiphar (Jakarta, Indonesia) for giving us paracetamol and PT. Konimex (Surakarta, Indonesia) for providing propyphenazone and caffeine standards.

\section{REFERENCES}

Bozdoğan A., Acar AM., Kunt GK., 1992. Simultaneous determination of acetaminophen and caffeine in tablet preparations by partial least-squares multivariate spectrophotometric calibration. Talanta 39(8): 977-979.

Dimitrovskat A., Trajkovic-Jolevskat S., Nancovskat A., Ilievska M., 1995. Determination of propyphenazone, paracetamol, caffeine and codeine phosphate with thin layer chromatography. Bull. Chem. Technol. Macedonia, 14: 39 - 41.

Dinc, E., 1999. A comparative study of the ratio spectra derivative spectrophotometry, Vierordt's method and highperformance liquid chromatography applied to the simultaneous analysis of caffeine and paracetamol in tablets. $J$. Pharm. Biomed. Anal., 21(4): 723-730.

Dinc, E., Onur F., 1995. Application of a new spectrophotometric method for the analysis of a ternary mixture containing metamizol, paracetamol and caffeine in tablets. Anal. Chim. Acta, 359(1-2):93-106.

Domínguez Vidal A., Ortega Barrales P., Molina Díaz A. , 2003. Simultaneous Determination of Paracetamol, Caffeine and Propyphenazone in Pharmaceuticals by Means of a Single Flow-Through UV Multiparameter Sensor. Microchim. Acta, 141(3): 157-163.

Emre D., Ozaltin N., 2007. Simultaneous determination of paracetamol, caffeine and propyphenazone in ternary mixtures by micellar electrokinetic capillary chromatography. J. Chromatogr. B., 847(2):126-132.

Kiersch TA., Minic MR., 2002. The onset of action and the analgesic efficacy of Saridon ${ }^{\circledR} \quad$ (a propyphenazone/ paracetamol/ caffeine combination) in comparison with paracetamol, ibuprofen, aspirin and placebo (pooled statistical analysis). Curr. Med. Res. Opin., 18(1): 1825. 
Markoviz S., Kusez Z., 1992. Determination of butalbital, caffeine and propyphenazone in pharmaceutical preparations by gas chromatography method. Pharmazie 46(12): 886.

Miller, JN., Miller JC., 2005. Statistics and Chemometrics for Analytical Chemistry. 5th Edn., Pearson Education Ltd., England, pp: 213-239.

Mohamed AEI., Mikre W., 2009. Determination of lamivudine and stavudine in pharmaceutical preparations using chemometrics-assisted spectrophotometry. Saudi Pharm. J., 17: 275-281.

Roggo, Y., Chalus, P., Maurer, L., LemaMartinez C., Edmond A., Jent N., 2007. A Review of near infrared spectroscopy and chemometrics in pharmaceutical technologies. J. Pham. Biomed Anal. 44: $683-700$.

Rohman, A., 2012. Application of fourier transform infrared spectroscopy for quality control of pharmaceutical products: a review. Indonesian J. Pharm. 23(1): $1-8$.
Rohman, A., Man YBC., 2011. Application of Fourier transform infrared (FT-IR) spectroscopy combined with chemometrics for authentication of codliver oil. Vibrat. Spectroscopy, 55: 141-145.

Rohman, A., Silawati D., Sudjadi, Riyanto S., 2015. Simultaneous determination of sulfamethoxazole and trimethoprim using UV spectroscopy in combination with multivariate calibration. J. Med. Sci., 15: 178-184.

Soponar F., Staniloae D., Moise G., Szaniszlo B., David V., 2013. Simultaneous determination of paracetamol, propyphenazone and caffeine from pharmaceutical preparations in the presence of related substances using a validated HPLC-DAD method. Revue Roumaine de Chimie Rev. Roum. Chim., 58(4-5): 433-440.

Wold S., Trygg J., Berglund A., Antti H., 2001. Some recent developments in PLS modeling. Chemom. Intell. Lab. Syst. 58: 131-150. 